\title{
Prospects of inflation in delicate D-brane cosmology
}

\author{
Sudhakar Panda, ${ }^{1}$ M. Sami, ${ }^{2}$ and Shinji Tsujikawa ${ }^{3}$ \\ ${ }^{1}$ Harish-Chandra Research Institute, Chhatnag Road, Jhusi, Allahabad-211019, Indid \\ ${ }^{2}$ Centre of Theoretical Physics, Jamia Millia Islamia, New Delhi-110025, Indid \\ ${ }^{3}$ Department of Physics, Gunma National College of Technology, Gunma 371-8530, Japan
}

(Dated: November 8, 2018)

\begin{abstract}
We study D-brane inflation in a warped conifold background that includes brane-position dependent corrections for the nonperturbative superpotential. Instead of stabilizing the volume modulus $\chi$ at instantaneous minima of the potential and studying the inflation dynamics with an effective single field (radial distance between a brane and an anti-brane) $\phi$, we investigate the multi-field inflation scenario involving these two fields. The two-field dynamics with the potential $V(\phi, \chi)$ in this model is significantly different from the effective single-field description in terms of the field $\phi$ when the field $\chi$ is integrated out. The latter picture underestimates the total number of e-foldings even by one order of magnitude. We show that a correct single-field description is provided by a field $\psi$ obtained from a rotation in the two-field space along the background trajectory. This model can give a large number of e-foldings required to solve flatness and horizon problems at the expense of fine-tunings of model parameters. We also estimate the spectra of density perturbations and show that the slow-roll parameter $\eta_{\psi \psi}=M_{\mathrm{pl}}^{2} V_{, \psi \psi} / V$ in terms of the rotated field $\psi$ determines the spectral index of scalar metric perturbations. We find that it is generally difficult to satisfy, simultaneously, both constraints of the spectral index and the COBE normalization, while the tensor to scalar ratio is sufficiently small to match with observations.

PACS numbers: $98.80 . \mathrm{Cq}$
\end{abstract}

\section{INTRODUCTION}

Modern Cosmology witnessed a revolution in 1980 with the advent of cosmological inflation [1]. The paradigm has stood the test of theoretical and observational challenges in the past two decades [2, 3]. In spite of its cosmological successes to solve horizon and flatness problems, it still remains a paradigm in search of a viable theoretical model. It is, therefore, not surprising that efforts are underway to derive inflationary models from string theory, a consistent quantum field theory around the Planck's scale. The discovery of nonperturbative solutions, in string theory, called D-branes [4] has given rise to new hopes in this direction.

The D-brane cosmology is a subject of intense study at present. It ranges from inflation on a non-BPS brane because of tachyon condensation to inflation due to the motion of a D3-brane towards an anti-D3-brane [5, 6, 7, 8]. Efforts also have been made to study inflation due to geometric tachyon arising from the motion of a probe brane in the background of a stack of either NS5branes or the dual D5-branes [9] . These models are constructed in the framework of effective field theory and assume an underlying mechanism for the stabilization of various moduli fields.

An important step towards a realistic inflationary model in string theory emerged from the realization that background fluxes can stabilize most of the modulus

\footnotetext{
*Electronic address: panda@mri.ernet.in

$\dagger$ Electronic address: sami@jamia-physics.net

${ }^{\ddagger}$ Electronic address: shinji@nat.gunma-ct.ac.jp
}

fields. As shown in Ref. [10], the fluxes in a warped compactification, using a Klebanov-Strassler (KS) throat [1], can stabilize the dilaton and complex structure moduli of type IIB string theory compactified on an orientifold of a Calabi-Yau threefold. In fact it was demonstrated in Ref. [12] that all the closed string moduli can be stabilized by a combination of fluxes and nonperturbative effects. The nonperturbative effects are mainly responsible for stabilizing the Kähler moduli; they arise, via gauge dynamics of either an Euclidean D3brane or from a stack of $n$ D7-branes wrapping supersymmetrically a four cycle in the warped throat. The warped volume of the four cycle controls the magnitude of the nonperturbative effect since it affects the gauge coupling on the D7-branes wrapping this four cycle.

The above formalism could lead to the construction of a realistic inflationary model [13], in string theory, which is built upon the compactification data (see also Refs. [14]). The inflaton potential can be obtained by performing string theoretic computations involving the details of the compactification scheme. In this setup inflation is realized by the motion of a D3-brane towards a distant static anti-D3-brane, placed at the tip of the throat. The position of the moving brane in the compactification manifold is identified with the inflaton field. To be more precise, the location of the mobile brane can be labeled with five angular coordinates and one radial coordinate, $r$. The canonical inflaton field $\phi$ is expressed by a constant re-scaling of this radial coordinate.

In Ref. [15], the embedding of the D7-branes as given in [16] was considered. It was assumed that at least one of the four-cycles carrying the nonperturbative effects descend down a finite distance into the warped throat. It 
was then shown that the presence of a D3-brane gives rise to a perturbation to the warp factor affecting a correction to the warped four cycle volume. Moreover, this correction depends on the position of the D3-brane and thus the superpotential for the nonperturbative effect gets corrected by an overall position-dependent factor. The total potential, that the mobile D3-brane experiences, is the sum of the potential (F-term) coming from the superpotential and the usual D-term potential contributed by the interaction between the D3-brane and the antiD3-brane. When the corrections to the nonperturbative superpotential is taken into account, the issue of volume modulus stabilization needs to be re-analyzed. This has been carried out in Refs. [17, 18, 19] and the viability of inflation was investigated in this modified scenario.

The model in Ref. [17, 18] is described by a two-field potential $V(\phi, \sigma)$ in terms of the inflaton $\phi$ and the volume modulus $\sigma$. If the mass of the modulus is much larger than the Hubble rate, the field $\sigma$ approximately evolves along the instantaneous minima determined by the condition $V_{, \sigma}=0$. One can obtain an effective singlefield potential with respect to $\phi$ by integrating out $\sigma$ in this way. However, we need to be careful for the fact that the actual trajectory is determined by the direction along the velocity of the fields. We shall introduce a new rotated field $\psi$ along the background trajectory and show that the usual single-field description holds for $\psi$ and not for $\phi$. As a result the single-field description in terms of $\phi$ underestimates the total amount of e-foldings even by one order of magnitude. This reflects that fact that the amount of inflation is sensitive to the slight change of the potential and also of model parameters.

We shall also calculate the spectra of density perturbations generated in this model. Again the spectral index $n_{\mathcal{R}}$ is determined by the slow-roll parameter $\eta_{\psi \psi}=M_{\mathrm{pl}}^{2} V_{, \psi \psi} / V$ instead of $\eta_{\phi \phi}=M_{\mathrm{pl}}^{2} V_{, \phi \phi} / V$. Thus we show that the correct single-field description in terms of $\psi$ is crucially important to study the perturbation spectra as well as the background dynamics.

\section{THE D3-BRANE POTENTIAL}

In this section we review the derivation of the scalar potential [17, 18] on a mobile D3-brane. The fluxes for the compactification of type IIB string theory on an orientifold of Calabi-Yau theory are chosen such that the internal space has a warped throat region. For example, the local geometry is taken to be the warped deformed conifold which is a subspace of complex dimension three in a four dimensional complex space defined by the constraint $\sum_{i=1}^{4} z_{i}^{2}=\epsilon^{2}$ where $z_{i}$ are coordinates in the four dimensional complex space and $\epsilon$ is real and corresponds to the deformation parameter. When the region of relevance for the D-brane inflation is chosen to lie far from the tip of the throat, the deformed parameter can be neglected. We can then choose $z_{\alpha}=\left(z_{1}, z_{2}, z_{3}\right)$ as the three independent complex coordinates for the position of the D3-brane (open string moduli) and use the conifold constraint to express $z_{4}$ in terms of these three coordinates. The throat can be glued into a compact space, which is assumed to have a single Kähler modulus $\rho$.

One can take the Kähler potential to be

$$
k=\frac{3}{2}\left(\sum_{i=1}^{4}\left|z_{i}\right|^{2}\right)^{2 / 3}=\frac{3}{2} r^{2}=\hat{r}^{2},
$$

so that the Kähler metric on the conifold, $k_{\alpha, \bar{\beta}} \equiv \partial_{\alpha} \partial_{\bar{\beta}} k$, is Ricci-flat. Thus the metric of the deformed conifold can be written as $\mathrm{d} s_{6}^{2}=\mathrm{d} \hat{r}^{2}+\hat{r}^{2} \mathrm{~d} s_{T_{1,1}^{1,1}}^{2}$, where $\mathrm{d} s_{T^{1,1}}^{2}$ is the metric on the Einstein space $T^{1,1}$, which can be expressed in terms of five angular coordinates. These angular coordinates and the real radial coordinate $r$ are the basis for the complex coordinates $z_{i}$, see Ref. [18] for details.

The $N=1$ supergravity F-term scalar potential involving the DeWolfe-Giddings Kähler potential $\mathcal{K}$ and the super potential $W$ is given by

$$
V_{F}=e^{\kappa^{2} \mathcal{K}}\left[D_{\Sigma} W \mathcal{K}^{\Sigma \bar{\Gamma}} \bar{D}_{\Gamma} W-3 \kappa^{2} W \bar{W}\right],
$$

where $D_{\Sigma} W=\partial_{\Sigma} W+\kappa^{2}\left(\partial_{\Sigma} \mathcal{K}\right) W, \quad\left\{Z^{\Sigma}\right\} \equiv$ $\left\{\rho, z_{\alpha} ; \alpha=1,2,3\right\}$ and $\kappa^{2}=M_{\mathrm{pl}}^{-2} \equiv 8 \pi G$. The total Kähler potential $\mathcal{K}$ depends upon $\rho$ and the open string moduli $z_{\alpha}$, and is given by [20]

$$
\begin{aligned}
\mathcal{K}\left(\rho, \bar{\rho}, z_{\alpha}, \bar{z}_{\alpha}\right) & =-3 M_{\mathrm{pl}}^{2} \ln \left[\rho+\bar{\rho}-\gamma k\left(z_{\alpha}, \bar{z}_{\alpha}\right)\right] \\
& \equiv-3 M_{\mathrm{pl}}^{2} \ln U
\end{aligned}
$$

where $k\left(z_{\alpha}, \bar{z}_{\alpha}\right)$ is the little Kähler potential, as defined in Eq. (11), for the metric on the conifold. The parameter $\gamma$ is proportional to the ratio of warped volumes of four cycle (before the D3-brane enters the throat) and the three-fold. In the expression (2) for the scalar potential, $\mathcal{K}^{\Sigma \bar{\Gamma}}$ is the inverse Kähler metric which can be derived from the Kähler potential $\mathcal{K}$. This leads to the following result 


$$
\begin{aligned}
V_{F}\left(\rho, z_{\alpha}\right)=\frac{\kappa^{2}}{3 U^{2}}\left[\left\{\rho+\bar{\rho}+\gamma\left(k,{ }_{\alpha} k^{\alpha \bar{\beta}} k, \bar{\beta}-k\right)\right\}\left|W,_{\rho}\right|^{2}-3(\bar{W} W, \rho+c . c)\right. \\
\left.+\left(k^{\alpha \bar{\beta}} k, \bar{\beta} \bar{W},_{\rho} W, \alpha+c . c\right)+\frac{1}{\gamma} k^{\alpha \bar{\beta}} W,_{\alpha} \bar{W},_{\beta}\right] .
\end{aligned}
$$

The first line in Eq. (4) is the standard Kachru-KalloshLinde-Trivedi (KKLT) F-term potential [12], while the rest of terms owe their existence entirely to the dependence of the nonperturbative superpotential on the open string moduli [15] (see also Refs. 21]).

This expression simplifies further, when we make use of the conifold metric (and its inverse) computed from the Kähler potential given in Eq. (1), to

$V_{F}\left(\rho, z_{\alpha}\right)=\frac{\kappa^{2}}{3 U^{2}}\left[(\rho+\bar{\rho})\left|W,_{\rho}\right|^{2}-3(\bar{W} W, \rho+c . c)+.\frac{3}{2}\left(z_{\alpha} \bar{W},{ }_{\rho} W, \alpha+c . c.\right)+\frac{1}{\gamma} r\left(\delta^{\alpha \beta}+\frac{1}{2} \frac{z_{\alpha} \bar{z}_{\beta}}{r^{3}}-\frac{z_{\beta} \bar{z}_{\alpha}}{r^{3}}\right) W,{ }_{, \alpha} \bar{W}, \beta\right]$

Note that the expression for $U$, now, is simply $U(\rho, r)=$ $\rho+\bar{\rho}-(3 / 2) \gamma r^{2}$. To obtain the full potential, we add to (5), the contribution of an anti-D3-brane at the tip of the conifold, including its Coulomb interaction with the moving D3-brane [12]:

$$
V_{D}(\rho, r)=\frac{D_{0}}{U^{2}(\rho, r)}\left[1-\frac{3 D_{0}}{16 \pi^{2}\left(T_{3} r^{2}\right)^{2}}\right]
$$

where $D_{0} \equiv 2 h_{0}^{-1} T_{3}$ is twice the warped D3-brane tension at the tip of the throat. The total potential on the mobile brane is thus given by $V=V_{F}\left(\rho, z_{\alpha}\right)+V_{D}(\rho, r)$.

The superpotential $W=W_{0}+W_{\text {np }}$ where $W_{0}$ is the Gukov-Vafa-Witten flux super potential [22] and $W_{\mathrm{np}}=$ $A\left(z_{\alpha}\right) e^{-b \rho}$ is the contribution from the nonperturbative effects. Here the factor, $b \equiv 2 \pi / n$, arises from gauge dynamics on a stack of $n$ number of D7-branes. These D7-branes wrap a four-cycle in the warped throat preserving supersymmetry which is specified by the embedding equation $f\left(z_{\alpha}\right)=0$. The presence of the D3-brane also leads to a perturbation to the warp factor which results in a correction to the warped four-cycle volume.
This correction has been found to be D3-brane position dependent and, in fact, is responsible for the pre-factor $A\left(z_{\alpha}\right)$ in $W_{\text {np }}$ [23]. In Ref. [15] this position dependence is found to be

$$
A\left(z_{\alpha}\right)=A_{0}\left(\frac{f\left(z_{\alpha}\right)}{f(0)}\right)^{1 / n}
$$

where $A_{0}$ is related to the threshold corrections which depend on complex structure moduli and for us it is just a constant parameter as these are already stabilized by the flux background. Note that the relation (7) can be derived generically without specifying the embeddings in the warped deformed conifold 24]. The potential, $V\left(\rho, r, z_{i}\right)$, thus is a complicated function of the Kähler modulus, the radial and the five angular coordinates of the mobile D3-brane. However, the angular coordinates can be integrated out as mentioned below.

Choosing the holomorphic embedding of Ref. [16], given by the equation $f\left(z_{1}\right)=\mu-z_{1}=0$, we have $A\left(z_{1}\right)=A_{0}\left(1-z_{1} / \mu\right)^{1 / n}$. Using this and also setting $\rho=\sigma+i \tau$ in Eq. (5) we find

$$
V_{F}=\frac{\kappa^{2} b|A|^{2} e^{-2 b \sigma}}{3 U^{2}}\left[2 b \sigma+6+6 W_{0} e^{b \sigma} \operatorname{Re}\left(\frac{e^{i b \tau}}{A}\right)+\frac{3}{2 n} \frac{\mu\left(z_{1}+\bar{z}_{1}\right)-2\left|z_{1}\right|^{2}}{\left|\mu-z_{1}\right|^{2}}+\frac{r}{b \gamma}\left(1-\frac{\left|z_{1}\right|^{2}}{2 r^{3}}\right) \frac{1}{n^{2}\left|\mu-z_{1}\right|^{2}}\right]
$$

Note that this potential has only one term that depends on $\tau$. The potential for $\tau$ is minimized when this term takes the smallest possible value. Since the coefficient of this term contains $W_{0}$ which is negative, integrating out this field amounts to replacing $e^{i b \tau} / A$ by $|A|^{-1}$. Similarly, the five angular coordinates, which describe the position of the D3-brane on the base of the throat, are periodic coordinates on a compact space. Thus, the potential in 
these fields is either constant or has discrete minima for some fixed values of these five fields. Since the radial motion of the brane is of special interest, we can focus on the stable trajectories in the angular directions which minimize the potential.

In the case of the embedding $f\left(z_{1}\right)=0$, the stable minima in angular directions occur only for trajectories that obey $z_{1}=-r^{3 / 2} / \sqrt{2}[18]$. From the DBI action of D3-brane, one can read out that the canonical field is $\phi=\sqrt{3 T_{3} / 2} r$, which corresponds to the approximation (when compared to the result obtained from the Kähler potential) that $\rho+\bar{\rho} \gg\left(\gamma / T_{3}\right) \phi^{2}$ and that the field $\sigma$ does not change much. Using this expression for $z_{1}$ in Eq. (8) and defining the minimal radial coordinate (position) of the D7-brane embedding to be $r_{\mu}^{3} \equiv 2 \mu^{2}$ i.e., $\phi_{\mu}^{2}=(3 / 2) T_{3}\left(2 \mu^{2}\right)^{2 / 3}$, we can write the full potential, involving only two fields, the field $\phi$ and the volume modulus $\sigma$, in the following form

$$
\begin{aligned}
V(\phi, \sigma)= & \frac{b\left|A_{0}\right|^{2}}{3 M_{\mathrm{pl}}^{2}} \frac{e^{-2 b \sigma}}{U^{2}(\phi, \sigma)} g^{2 / n}(\phi)\left[2 b \sigma+6-6 e^{b \sigma} \frac{\left|W_{0}\right|}{\left|A_{0}\right|} \frac{1}{g^{1 / n}(\phi)}+\frac{3}{n}\left\{c \frac{\phi}{\phi_{\mu}}-\left(\frac{\phi}{\phi_{\mu}}\right)^{3 / 2}-\left(\frac{\phi}{\phi_{\mu}}\right)^{3}\right\} \frac{1}{g^{2}(\phi)}\right] \\
& +\frac{D(\phi)}{U^{2}(\phi, \sigma)}
\end{aligned}
$$

where

$$
\begin{aligned}
U(\phi, \sigma) & =2 \sigma-\frac{\gamma}{T_{3}} \phi^{2}, \\
g(\phi) & =1+\left(\frac{\phi}{\phi_{\mu}}\right)^{3 / 2}, \\
D(\phi) & =D_{0}\left(1-\frac{27 D_{0}}{64 \pi^{2} \phi^{4}}\right),
\end{aligned}
$$

and $c=1 /\left(6 \pi \gamma T_{3} \phi_{\mu}^{2}\right)$.

We will use this form of the two-field potential in our analysis in the next section keeping in mind that $A_{0}$ and $W_{0}$ have the dimension $[\text { mass }]^{3}$ while for $D_{0}$ it is $[\text { mass }]^{4}$. It should be noted that the field $\sigma$ is not yet in the canonical form. From the Kähler potential (3), we find that the kinetic term, $-K_{\rho \bar{\rho}} \partial_{\mu} \rho \partial^{\mu} \bar{\rho}$, becomes canonical (at the tip of the throat, which coincides with the same approximation made for the canonical field $\phi$ ) if we consider the field $\chi$ defined by

$$
\frac{\chi}{M_{\mathrm{pl}}}=\sqrt{\frac{3}{2}} \ln \sigma
$$

In what follows we shall examine the viability of inflation in the frame work of two-field dynamics described by the potential $V(\phi, \chi)$. This potential amounts to replacing $\sigma$ in Eq. (9) by $\exp \left(\sqrt{2 / 3} \chi / M_{\mathrm{pl}}\right)$.

\section{BACKGROUND EVOLUTION}

In this section we discuss the dynamics of inflation induced by the D3-brane potential (9) but with the canonical field $\chi$ replacing the field $\sigma$. In the flat FriedmannRobertson-Walker (FRW) metric with a scale factor $a$ the equations of motion are given by

$$
\begin{aligned}
& \dot{H}=-\frac{1}{2 M_{\mathrm{pl}}^{2}}\left(\dot{\phi}^{2}+\dot{\chi}^{2}\right), \\
& \ddot{\phi}+3 H \dot{\phi}+V_{, \phi}=0, \\
& \ddot{\chi}+3 H \dot{\chi}+V_{, \chi}=0,
\end{aligned}
$$

together with the constraint equation

$$
3 H^{2}=\frac{1}{M_{\mathrm{pl}}^{2}}\left[\frac{1}{2} \dot{\phi}^{2}+\frac{1}{2} \dot{\chi}^{2}+V(\phi, \chi)\right],
$$

where a dot represents a derivative with respect to cosmic time $t$ and $H \equiv \dot{a} / a$ is the Hubble parameter. For later convenience we introduce the following slow-roll parameters

$$
\begin{aligned}
& \epsilon_{\phi}=\frac{M_{\mathrm{pl}}^{2}}{2}\left(\frac{V_{, \phi}}{V}\right)^{2}, \quad \epsilon_{\chi}=\frac{M_{\mathrm{pl}}^{2}}{2}\left(\frac{V_{, \chi}}{V}\right)^{2}, \\
& \eta_{\phi \phi}=M_{\mathrm{pl}}^{2} \frac{V_{, \phi \phi}}{V}, \quad \eta_{\chi \chi}=M_{\mathrm{pl}}^{2} \frac{V_{, \chi \chi}}{V}, \quad \eta_{\phi \chi}=M_{\mathrm{pl}}^{2} \frac{V_{, \phi \chi}}{V} .
\end{aligned}
$$

The squared masses of the fields $\phi$ and $\chi$ are defined by $m_{\phi \phi}^{2} \equiv V_{, \phi \phi}$ and $m_{\chi \chi}^{2} \equiv V_{, \chi \chi}$, respectively. Since the Hubble parameter approximately satisfies the relation $3 H^{2} \simeq V / M_{\mathrm{pl}}^{2}$ during inflation, the slow-roll parameters $\eta_{\phi \phi}$ and $\eta_{\chi \chi}$ are given by

$$
\eta_{\phi \phi} \simeq \frac{m_{\phi}^{2}}{3 H^{2}}, \quad \eta_{\chi \chi} \simeq \frac{m_{\chi}^{2}}{3 H^{2}} .
$$

Since we are considering the case in which the field $\phi$ plays the role of the inflaton, we require that $\eta_{\phi \phi}$ is not much larger than unity. Meanwhile the modulus field $\chi$ can be heavy $\left(\eta_{\chi \chi} \gtrsim 1\right)$ or light $\left(\eta_{\chi \chi} \lesssim 1\right)$ depending 


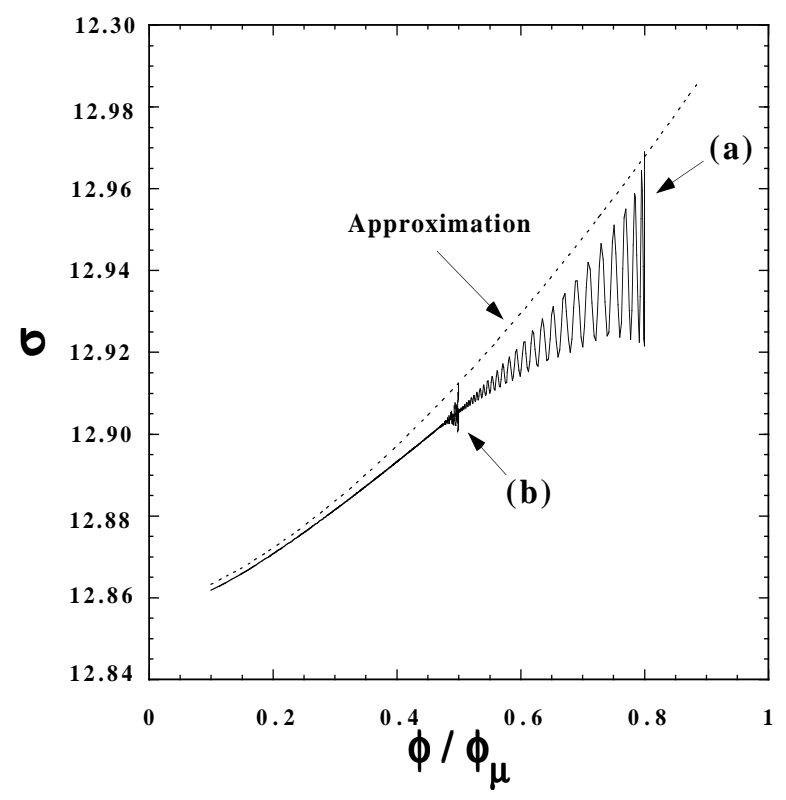

FIG. 1: The trajectory of the scalar fields in the $(\phi, \sigma)$ plane for the model parameters $n=8, A_{0}=1, \omega_{0} \equiv b \sigma_{0}=10.1$, $\omega_{F}=9.9951, W_{0}=3.496 \times 10^{-4}, D_{0}=1.215 \times 10^{-8}$ and $\phi_{\mu}=0.25$. The curves (a) and (b) correspond to the trajectories derived by solving the background equations numerically for the initial conditions $\phi_{i} / \phi_{\mu}=0.8$ and $\phi_{i} / \phi_{\mu}=0.5$, respectively. The initial values of the field $\chi$ are chosen to satisfy the relation (20) with $\sigma_{0}=\omega_{0} / b=12.8597$. We also plot the approximate trajectory (20).

on the model parameters. The latter corresponds to the situation in which two stages of inflation can be realized.

When the $\chi$ mass is much larger than the Hubble rate $\left(\eta_{\chi \chi} \gg 1\right)$, the field $\chi$ rapidly rolls down toward instantaneous minima of the potential given by $V_{, \chi}=0$. In this case it was shown in Ref. [17, 18] that the evolution of the non-canonical field $\sigma=\exp \left(\sqrt{2 / 3} \chi / M_{\mathrm{pl}}\right)$ is approximately described by

$$
\sigma_{*} \approx \sigma_{0}\left[1+c_{3 / 2}\left(\frac{\phi}{\phi_{\mu}}\right)^{3 / 2}\right] .
$$

We note that this relation was also derived in Ref. [19]. Here $\sigma_{0}=3 \gamma M_{\mathrm{pl}}^{2} / T_{3}$ and $c_{3 / 2}=\left(1-1 / 2 \omega_{F}\right) / n \omega_{F}$, where $\omega_{F}$ satisfies the relation $3 e^{\omega_{F}}\left|W_{0}\right| /\left|A_{0}\right|=2 \omega_{F}+3$. The condition, $\omega_{F} \gg 1$, is assumed to reach the expression (20). We also note that the approximation (20) is not accurate in the large $\phi / \phi_{\mu}$ region close to 1 .

In Fig. 1 we illustrate a typical example for the trajectory of the two fields in the $(\phi, \sigma)$ plane. The case (a) corresponds to the initial conditions $\phi_{i} / \phi_{\mu}=0.8$ and $\sigma_{i}$ satisfying Eq. (20). Since the approximation (20) is not accurate in the large $\phi / \phi_{\mu}$ region, the initial position of the field $\sigma$ does not exactly match with the local minimum of the potential $\left(V_{, \chi}=0\right)$. The field $\sigma$ quickly oscillates around instantaneous minima of the potential at the initial stage. In this case the system enters the slow-roll inflation stage around $\phi / \phi_{\mu} \lesssim 0.5$ after the field $\chi$ is almost stabilized at the instantaneous minima. If we choose smaller initial $\phi / \phi_{\mu}$ (such as the case (b) in Fig. (1), the period of the oscillation of $\chi$ becomes very short. Figure 1 shows that the accuracy of the approximation (20) becomes better for smaller values of $\phi / \phi_{\mu}$.

In the upper panel of Fig. 2 we plot the potential $V(\phi, \chi)$ as a function of $\phi / \phi_{\mu}$ for the same model parameters as in Fig. 1. Note that the field $\phi$ is obtained by projecting a two-field trajectory into the $\phi$ direction. Since $\eta_{\chi \chi}$ is of order $10^{3}$ in this case, the mass of the field $\chi$ is much larger than the Hubble parameter. When $\chi / M_{\mathrm{pl}}>3.141$ the potential does not possess instantaneous minima in the region $0<\phi / \phi_{\mu}<1$, but they appear for $\chi / M_{\mathrm{pl}}<3.141$. We require that $\chi / M_{\mathrm{pl}}$ is initially smaller than 3.160 for $\phi_{i} / \phi_{\mu}=0.8$ in order to avoid that the field evolves toward the forbidden region $\phi / \phi_{\mu}>1$.

The numerical simulation in Fig. 2 corresponds to the initial conditions $\phi_{i} / \phi_{\mu}=0.8$ and $\chi_{i} / M_{\mathrm{pl}}=3.1385$, which satisfy the relation (20). In this case the field $\phi$ slightly evolves toward larger $\phi$ at the initial stage, but it soon begins to roll down to instantaneous minima which move toward smaller $\phi$ with the decrease of $\chi$. From the lower panel of Fig. 2 2 we find that the field $\chi$ does not exist at the instantaneous minimum initially (because of the breakdown of the approximation (20) ) and then evolves toward the instantaneous minima with oscillations. The upper panel of Fig. 2 2 shows that the instantaneous minima in the $\phi$ direction disappear for $\chi / M_{\mathrm{pl}}<3.130$. This is the signal of the end of inflation. Thus the field $\chi$ does not evolve much during inflation in the above case, as required from the stabilization of the volume modulus. However, as we will see below, it is of crucial importance to incorporate the dynamics of the field $\chi$.

In Ref. [18] the authors reduce the two-field potential (9) to the single-field one by substituting the relation (20) for (9):

$$
V_{*}(\phi)=V\left(\phi, \sigma_{*}(\phi)\right) .
$$

In Fig. 3 we plot the numerically obtained potential $V$ as a function of $\phi / \phi_{\mu}$ to compare with (21). The potential in the two-field system is flatter than in the case derived under the single $\phi$ field approximation. This implies that the real two-field system chooses a trajectory which gives a larger amount of inflation.

In Fig. 4 we show the evolution of the number of efoldings $\ln a$ in terms of the function of $\phi / \phi_{\mu}$ with the initial conditions $\phi_{i} / \phi_{\mu}=0.8$ and $\dot{\phi}_{i} / \phi_{\mu}=-1.0 \times 10^{-10} \mathrm{~m}$, where $m=10^{-7} M_{\mathrm{pl}}$. In the two-field case inflation occurs around the region $0.3 \lesssim \phi / \phi_{\mu} \lesssim 0.5$, which leads to the number of e-foldings $\ln a=67$ at the end of inflation. We find that this is not sensitive to the change of initial conditions as long as $\phi_{i} / \phi_{\mu}$ is larger than 0.5. The change of initial velocities of scalar fields hardly affects the evolution of the slow-roll regime, unless we choose unnaturally large initial velocities. In the single $\phi$ field 


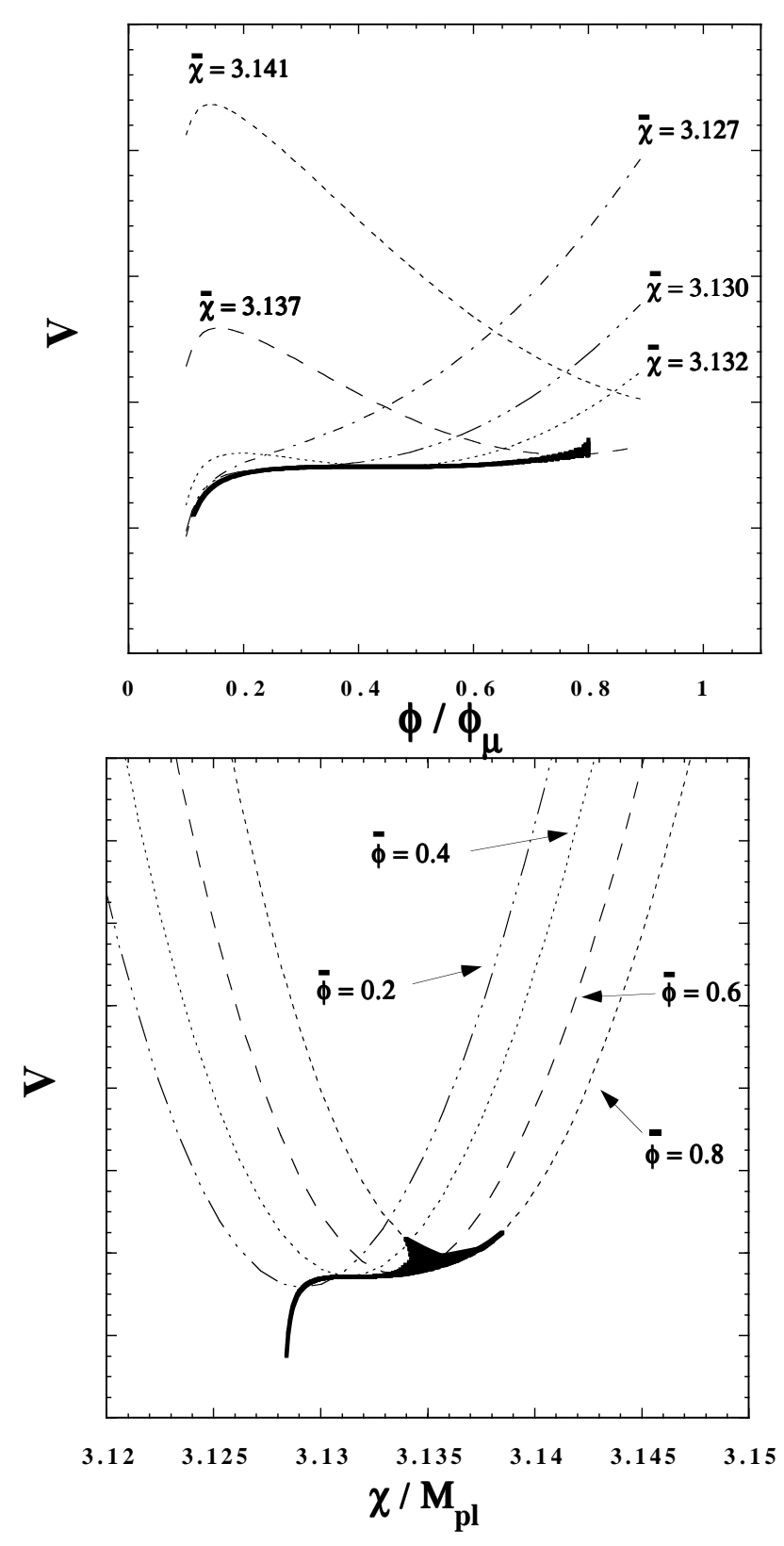

FIG. 2: The potential $V(\phi, \chi)$ for the model parameters $n=$ $8, A_{0}=1, \omega_{0} \equiv b \sigma_{0}=10.1, \omega_{F}=9.9951, W_{0}=3.496 \times 10^{-4}$, $D_{0}=1.215 \times 10^{-8}$ and $\phi_{\mu}=0.25$. The solid curves correspond to the one derived by solving the background equations numerically for the initial conditions $\phi_{i} / \phi_{\mu}=0.8$ and $\dot{\phi}_{i} / \phi_{\mu}=-1.0 \times 10^{-10} m$, where $m=10^{-7} M_{\mathrm{pl}}$ is a mass to normalize time $t$. Note that the initial conditions for the field $\chi$ are chosen to satisfy the relation (20), i.e., $\chi_{i} / M_{\mathrm{pl}}=3.1385$. The upper panel shows the potential in terms of the function of $\phi / \phi_{\mu}$ for several fixed values of $\bar{\chi} \equiv \chi / M_{\mathrm{pl}}$ (plotted as dotted curves). The lower panel shows the potential in terms of the function of $\chi / M_{\mathrm{pl}}$ for several fixed values of $\bar{\phi} \equiv \phi / \phi_{\mu}$.

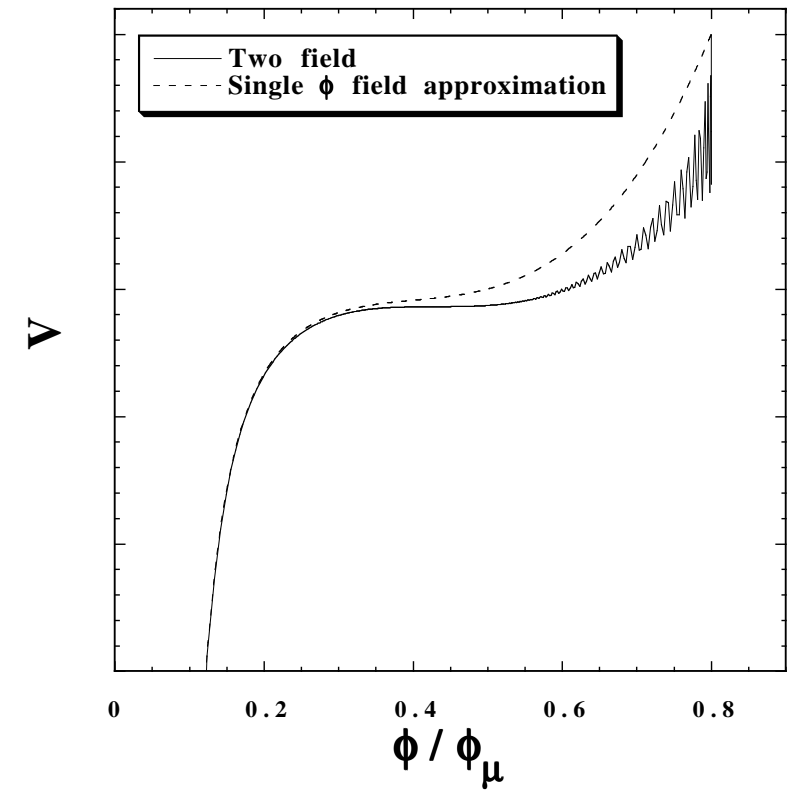

FIG. 3: The solid curve represents the potential obtained by numerically solving the background equations in two-field system for the same model parameters and initial conditions as in Fig. 22 The dotted curve corresponds to the potential (21) under the single $\phi$ field approximation.

approximation, compared to the two-field system, we obtain a much smaller value of the number of e-foldings: $\ln a=7$.

This discrepancy reflects the fact that the background trajectory along which the fields evolve is not given by the field $\phi$ but by the field $\psi$ satisfying the relation

$$
\dot{\psi}=(\cos \theta) \dot{\phi}+(\sin \theta) \dot{\chi},
$$

where

$$
\tan \theta=\dot{\chi} / \dot{\phi} .
$$

Here $\theta$ characterizes the direction along which the scalar fields evolve. If the field trajectory is curved as in our case then we have $\dot{\theta} \neq 0$. Since $\dot{s} \equiv-(\sin \theta) \dot{\phi}+(\cos \theta) \dot{\chi}=0$ from Eq. (23), the fields do not move to the direction orthogonal to $\psi$.

In order to find out an effective single-field trajectory we need to obtain the potential $V$ in terms of the function of $\psi$ rather than $\phi$. If the field $\chi$ is frozen at some particular value $(\chi=$ const), we just need to derive the potential with respect to $\phi$ because $\psi$ coincides with $\phi$. The hybrid inflation model with the potential $V=\frac{\lambda}{4}\left(\chi^{2}-M^{2} / \lambda\right)^{2}+\frac{1}{2} g^{2} \phi^{2} \chi^{2}+\frac{1}{2} m^{2} \phi^{2}$ [25] falls into this category: inflation occurs along the line $\chi=0$. However the model (9) gives a curved background trajectory, which means that the single-field description in terms of $\psi$ is necessary to understand the dynamics of inflation correctly.

The mass squared of the field $\psi$ is given by [26]

$$
V_{, \psi \psi}=\left(\cos ^{2} \theta\right) V_{, \phi \phi}+(\sin 2 \theta) V_{, \phi \chi}+\left(\sin ^{2} \theta\right) V_{, \chi \chi} .
$$




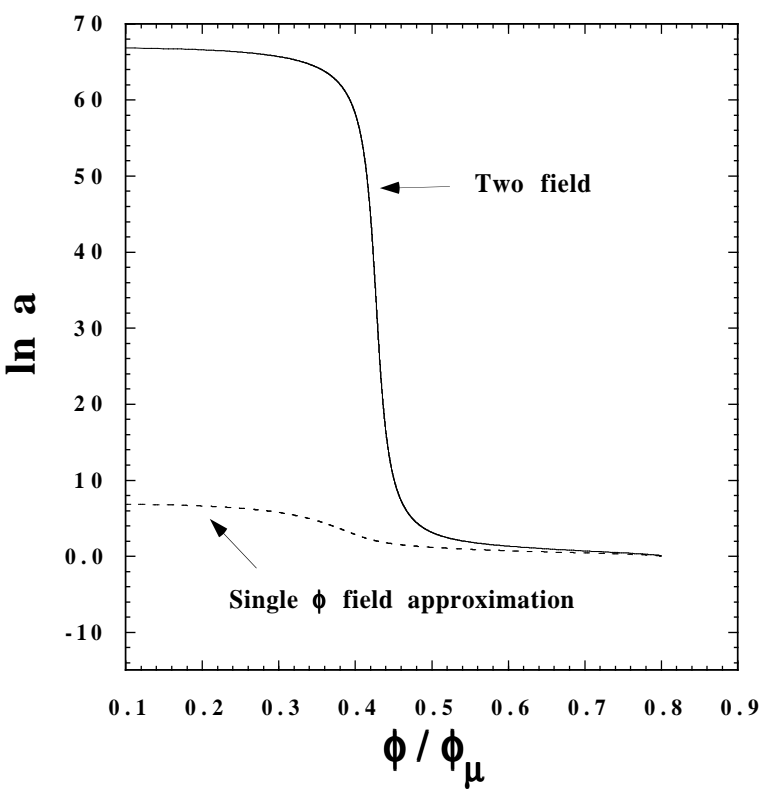

FIG. 4: The evolution of the number of e-foldings in terms of the function of $\phi / \phi_{\mu}$ for the two-field system (solid curve) and for the system under the single $\phi$ field approximation (dotted curve). The model parameters and initial conditions are the same as in Fig. 2

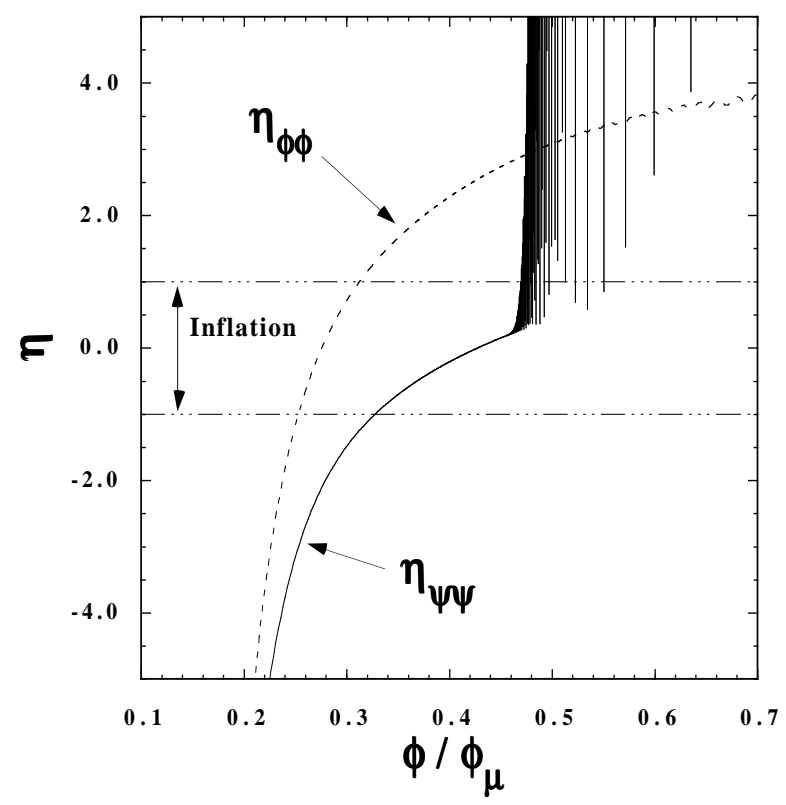

FIG. 5: The evolution of the slow-roll parameters $\eta_{\psi \psi}$ and $\eta_{\phi \phi}$ in terms of the function of $\phi / \phi_{\mu}$. The model parameters and initial conditions are chosen as in the case of Fig. 2 The period of inflation is determined by the condition $\left|\eta_{\psi \psi}\right|<1$.

Then the slow-roll parameter, $\eta_{\psi \psi} \equiv M_{\mathrm{pl}}^{2} V_{, \psi \psi} / V$, is

$$
\eta_{\psi \psi}=\left(\cos ^{2} \theta\right) \eta_{\phi \phi}+(\sin 2 \theta) \eta_{\phi \chi}+\left(\sin ^{2} \theta\right) \eta_{\chi \chi} .
$$

In Fig. 5 we plot the evolution of the slow-roll parameters $\eta_{\psi \psi}$ and $\eta_{\phi \phi}$ for the same model parameters and initial

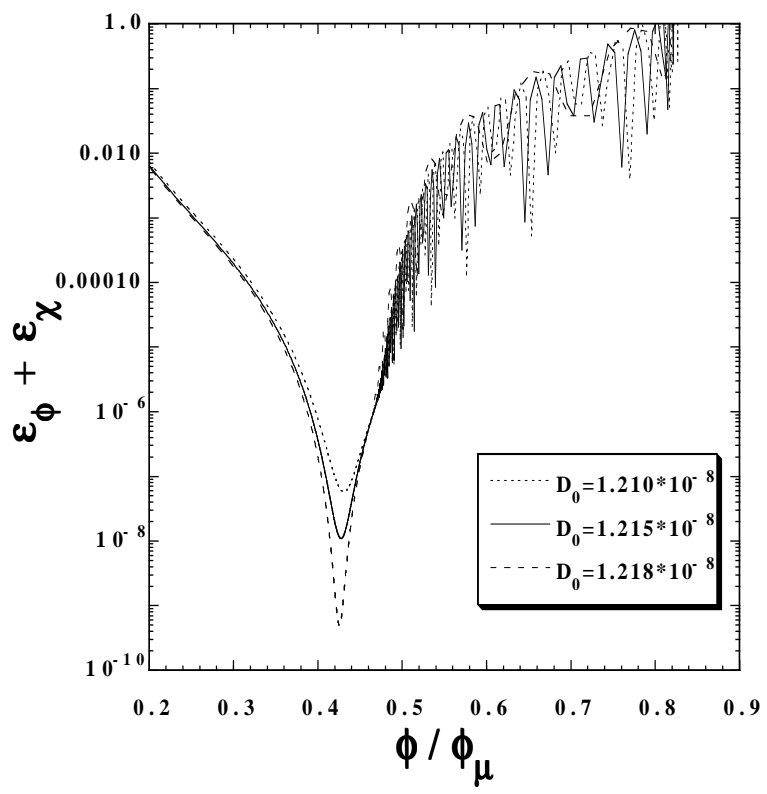

FIG. 6: The evolution of $\epsilon_{\phi}+\epsilon_{\chi}$ in terms of the function of $\phi / \phi_{\mu}$ with three different values of $D_{0}$. Other model parameters and initial conditions are chosen as in the case of Fig. 2 We find that $\epsilon_{\phi}+\epsilon_{\chi}$ are smaller than $10^{-4}$ during inflation $\left(0.3<\phi / \phi_{\mu}<0.5\right)$.

conditions as in Fig. 2. The period of the inflationary stage is determined by the condition $\left|\eta_{\psi \psi}\right|<1$ instead

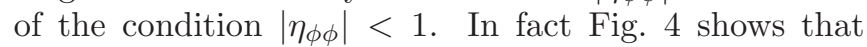
inflation occurs for $0.3 \lesssim \phi / \phi_{\mu} \lesssim 0.5$, which coincides with the region given by the condition $\left|\eta_{\psi \psi}\right|<1$ in Fig. 5 . The slow-roll parameter $\eta_{\phi \phi}$ is larger than unity during inflation, which implies that this variable is not suitable to describe the dynamics of inflation. In the next section we show that the slow-roll parameter $\eta_{\psi \psi}$, instead of $\eta_{\phi \phi}$, is crucially important to estimate the spectral index of scalar metric perturbations.

The scalar fields approach instantaneous minima of the potential so close that slow-roll parameters $\epsilon_{\phi}$ and $\epsilon_{\chi}$ become much smaller than $\left|\eta_{\psi \psi}\right|$ during inflation, see Fig. 6. It is possible to have a larger total number of e-foldings

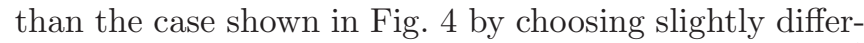
ent values of $D_{0}$. For example, when $D_{0}=1.218 \times 10^{-8}$ instead of $D_{0}=1.215 \times 10^{-8}$, we find that the total number of e-foldings becomes $\ln a=148$. In this case the field stays at instantaneous minima of the potential for a longer time. Then we obtain smaller values of $\epsilon_{\phi}$ and $\epsilon_{\chi}$, which leads to a larger amount of inflation. This behavior is clearly seen in the numerical simulation of Fig. 6. Inflation ends when the slow-roll parameter $\left|\eta_{\psi \psi}\right|$ becomes larger than unity. Meanwhile if we choose $D_{0}=1.210 \times 10^{-8}$ then the total number of e-foldings is found to be $\ln a=43$, which is not sufficient to solve horizon and flatness problems. When $D_{0}=1.220 \times 10^{-8}$ inflation does not end because the fields are stuck at the local minimum of the potential. These results show how the number of e-foldings is sensitive to the choice of model 
parameters. Thus we require a severe fine-tuning as it is typical in the context of D-brane inflation. In the field equations (15) and (16) we have dropped other kinetic terms coming from the Kähler potential which are suppressed by $\mathcal{O}\left(\phi / M_{\mathrm{pl}}^{2}\right)$. We have carried out numerical simulations by taking into account those terms and found that the total number of e-foldings is hardly changed (the difference of order 0.1 ). Thus the results are insensitive to the inclusion of such kinetic terms.

The dynamics of inflation is mainly affected by the changes of the model parameters $W_{0}$ and $D_{0}$. We have run our numerical code for many other cases by changing $W_{0}$ and $D_{0}$ and found that the inflationary dynamics discussed above is a rather generic feature when the field $\chi$ is massive $\left(\eta_{\chi \chi} \gg 1\right)$. The single $\phi$ field approximation using the potential (21) tends to underestimate the total amount of inflation. We have also considered the parameter regions in which the $\chi$ mass is smaller than the Hubble parameter $\left(\eta_{\chi \chi} \lesssim 1\right)$. In such cases, we numerically find that the field $\chi$ typically evolves toward larger values unlike the estimation given in Eq. (20). In this case the instantaneous minima of the potential tend to disappear in the region $0<\phi / \phi_{\mu}<1$, which implies a difficulty to obtain a large number of e-foldings. Hence in what follows we concentrate on the case where the mass of the field $\chi$ is much larger than the Hubble parameter.

\section{COSMOLOGICAL PERTURBATIONS}

In this section we discuss the spectra of density perturbations generated in the model with the potential (9). In two-field models of inflation the resulting density perturbations are generally different from those in a single-field model because of the presence of isocurvature (entropy) perturbations [27] (see Refs. [28, 29] for review). We denote the field perturbations in $\phi$ and $\chi$ as $\delta \phi$ and $\delta \chi$. Along and orthogonal to the background trajectory in two-field space it is convenient to carry out a field rotation [26]:

$$
\begin{aligned}
& \delta \psi \equiv(\cos \theta) \delta \phi+(\sin \theta) \delta \chi, \\
& \delta s \equiv-(\sin \theta) \delta \phi+(\cos \theta) \delta \chi,
\end{aligned}
$$

where $\theta$ is defined by Eq. (23). Here $\delta \psi$ and $\delta s$ correspond to adiabatic and entropy perturbations, respectively.

The perturbed spacetime about the FRW background is described by the line element

$$
\begin{aligned}
\mathrm{d} s^{2}= & -(1+2 A) \mathrm{d} t^{2}+2 a\left(\partial_{i} B-S_{i}\right) \mathrm{d} x^{i} \mathrm{~d} t \\
& +a^{2}\left[(1-2 \varphi) \delta_{i j}+2 \partial_{i j} E+h_{i j}\right] \mathrm{d} x^{i} \mathrm{~d} x^{j}
\end{aligned}
$$

where $A, B, E, \varphi$ are scalar metric perturbations and $h_{i j}$ is the tensor perturbation (we omit to write vector perturbations). The comoving curvature perturbation $\mathcal{R}$ and the isocurvature perturbation $\mathcal{S}$ are defined by

$$
\mathcal{R}=\varphi+\frac{H \delta \rho}{\dot{\rho}}, \quad \mathcal{S}=\frac{H(\dot{\phi} \delta \chi-\dot{\chi} \delta \phi)}{\dot{\phi}^{2}+\dot{\chi}^{2}}
$$

where $\delta \rho$ is the total density perturbation. Using the field perturbations (26) and (27) these are simply expressed as [26]

$$
\mathcal{R}=\frac{H \delta \psi_{\varphi}}{\dot{\psi}}, \quad \mathcal{S}=\frac{H \delta s}{\dot{\psi}}
$$

where $\delta \psi_{\varphi}=\delta \psi+\dot{\psi} \varphi / H$.

The each Fourier mode for $\delta \psi_{\varphi}$ and $\delta s$ satisfies the following equations of motion [26]

$$
\begin{aligned}
& \delta \ddot{\psi}_{\varphi}+3 H \delta \dot{\psi}_{\varphi}+\left[\frac{k^{2}}{a^{2}}+V_{, \psi \psi}-\dot{\theta}^{2}-\frac{1}{M_{\mathrm{pl}}^{2} a^{3}}\left(\frac{a^{3} \dot{\psi}^{2}}{H}\right)\right] \delta \psi_{\varphi} \\
& =2(\dot{\theta} \delta s)^{\cdot}-2\left(\frac{V_{, \psi}}{\dot{\psi}}+\frac{\dot{H}}{H}\right) \dot{\theta} \delta s, \\
& \ddot{\delta} s+3 H \dot{\delta} s+\left(\frac{k^{2}}{a^{2}}+V_{, s s}+3 \dot{\theta}^{2}\right) \delta s=\frac{\dot{\theta}}{\dot{\psi}} \frac{4 M_{\mathrm{pl}}^{2} k^{2}}{a^{2}} \Psi
\end{aligned}
$$

where $k$ is a comoving wavenumber, $\Psi=\varphi+a^{2} H(\dot{E}-$ $B / a), V_{, \psi \psi}$ is defined by (24) and

$$
V_{, s s}=\left(\sin ^{2} \theta\right) V_{, \phi \phi}-(\sin 2 \theta) V_{, \phi \chi}+\left(\cos ^{2} \theta\right) V_{, \chi \chi} .
$$

This shows that in the large-scale limit $(k \rightarrow 0)$ the entropy perturbation $\mathcal{S}$ evolves independently of the curvature perturbation, while the curvature perturbation $\mathcal{R}$ is sourced by the entropy perturbation as long as the trajectory is curved $(\dot{\theta} \neq 0)$ in the field space.

The background trajectories we discussed in the previous section correspond to the cases in which the field $\psi$ is light and the field $s$ is heavy relative to the Hubble parameter during inflation. Hence the mass term $V_{, \psi \psi}$ in Eq. (31) is smaller than the order of $H^{2}$, whereas $V_{\text {,ss }}$ is larger than $H^{2}$. When both $V_{. \psi \psi}$ and $V_{\text {,ss }}$ are smaller than $H^{2}$, it was shown in Ref. [30, 31] that at the Hubble radius crossing $(k=a H)$ the cross-correlation $\mathcal{C}_{\mathcal{R} S}$ between curvature and isocurvature perturbations is zero at lowest order in slow-roll (while it does not vanish at the first order). In our case $(\eta, s s \gg 1)$ the power spectrum of the entropy perturbation, $\mathcal{P}_{\delta s} \equiv \frac{k^{3}}{2 \pi^{2}}|\delta s|^{2}$, behaves in the usual way $\left(\mathcal{P}_{\delta s} \simeq(k / 2 \pi a)^{2}\right)$ for $k^{2} / a^{2} \gg V_{, s s}$, but after the mass term $V_{\text {,ss }}$ becomes larger than $k^{2} / a^{2}$ the entropy perturbation decreases more rapidly $\left(\mathcal{P}_{\delta s} \propto a^{-3}\right)$. This latter stage occurs even before the Hubble radius crossing (i.e., $V_{\text {,ss }}>k^{2} / a^{2}>H^{2}$ ), which generally leads to the weaker cross-correlation at $k=a H$ relative to the case of two light scalar fields studied in Refs. 30, 31].

We then neglect the interacting terms on the r.h.s. of Eq. (31) for the modes $k>a H$ and obtain the spectrum $\mathcal{P}_{\delta \psi_{\varphi *}} \simeq\left(H_{*} / 2 \pi\right)^{2}$ at the Hubble exit at lowest order in slow-roll of the field $\psi$ (in what follows we use the symbol * to represent the quantities at the Hubble radius crossing, $k=a H)$. Hence from Eq. (30) the power spectrum of the curvature perturbation at $k=a H$ is given by

$$
\mathcal{P}_{\mathcal{R}_{*}} \simeq\left(\frac{H^{2}}{2 \pi \dot{\psi}}\right)_{*}^{2} \simeq\left(\frac{V}{24 \pi^{2} \epsilon M_{\mathrm{pl}}^{4}}\right)_{*},
$$


where

$$
\epsilon \equiv \frac{M_{\mathrm{pl}}^{2}}{2}\left(\frac{V_{, \psi}}{V}\right)^{2} \simeq \epsilon_{\phi}+\epsilon_{\chi} .
$$

Here the last approximate equality holds under the slowroll approximation. Note that we used the relation $\dot{\psi}^{2} \simeq$ $(2 / 3) \epsilon V$ and $3 H^{2} \simeq V / M_{\mathrm{pl}}^{2}$.

One can describe the evolution of perturbations after the Hubble exit $(k<a H)$ by using a transfer matrix [30]

$$
\left(\begin{array}{c}
\mathcal{R} \\
\mathcal{S}
\end{array}\right)=\left(\begin{array}{cc}
1 & T_{\mathcal{R S}} \\
0 & T_{\mathcal{S S}}
\end{array}\right)\left(\begin{array}{l}
\mathcal{R}_{*} \\
\mathcal{S}_{*}
\end{array}\right)
$$

where $T_{\mathcal{R} S}$ characterizes the correlation between curvature and isocurvature perturbations. The dimensionless measure of correlation is defined by

$$
r_{c} \equiv \frac{T_{\mathcal{R S}}}{\sqrt{1+T_{\mathcal{R S}}^{2}}},
$$

which is in the range $\left|r_{c}\right| \leq 1$ by definition.

If the masses of two scalar fields $\phi$ and $\chi$ are small relative to the Hubble parameter, it was found in Refs. 32. that the correlation measure $\left|r_{c}\right|$ can be close to the order of 1 in several models of two-field inflation. This comes from the fact that the entropy perturbation $\delta s$ is not suppressed after the Hubble radius crossing. Meanwhile if one of the fields is heavy then the amplitude of $\delta s$ exponentially decreases $\left(|\delta s| \propto a^{-3 / 2}\right)$, which results in a very weak correlation $\left(\left|r_{c}\right| \ll 1\right)$. Since we are considering this latter situation for the model (9), it is a good approximation to neglect the correlation after the Hubble radius crossing.

Using this property, the power spectrum of the curvature perturbation at the end of inflation is given by

$$
\mathcal{P}_{\mathcal{R}} \simeq \mathcal{P}_{\mathcal{R}_{*}} \simeq\left(\frac{V}{24 \pi^{2} \epsilon M_{\mathrm{pl}}^{4}}\right)_{*},
$$

which holds for $\left|r_{c}\right| \ll 1$. When the correlation is strong, the r.h.s. of Eq. (38) is multiplied by the factor $1 /\left(1-r_{c}^{2}\right)$ [30]. The spectrum index of the curvature perturbation, $n_{\mathcal{R}} \equiv 1+\mathrm{d} \ln \mathcal{P}_{\mathcal{R}} / \mathrm{d} \ln k$, is given by

$$
n_{\mathcal{R}} \simeq 1-6 \epsilon+2 \eta_{\psi \psi},
$$

where the slow-roll parameters are evaluated at the Hubble exit.

The tensor perturbation $h_{i j}$ is decoupled from the scalar perturbation and is frozen after the Hubble radius crossing. Thus its power spectrum is given by [30]

$$
\mathcal{P}_{T}=\mathcal{P}_{T_{*}} \simeq \frac{2 V_{*}}{3 \pi^{2} M_{\mathrm{pl}}^{4}},
$$

with the spectral index

$$
n_{T} \equiv \frac{\mathrm{d} \ln P_{T}}{\mathrm{~d} \ln k}=-2 \epsilon .
$$

We also obtain the tensor to scalar ratio

$$
r \equiv \frac{\mathcal{P}_{T}}{\mathcal{P}_{\mathcal{R}}} \simeq 16 \epsilon \simeq 16\left(\epsilon_{\phi}+\epsilon_{\chi}\right),
$$

which is valid for $\left|r_{c}\right| \ll 1$. In the presence of the correlation the r.h.s. of Eq. (42) is multiplied by the factor $\left(1-r_{c}^{2}\right)$ 30, 33].

As we mentioned in the previous section, the slowroll parameters $\epsilon_{\phi}$ and $\epsilon_{\chi}$ for the model (9) are typically very much smaller than 1 because the scalar fields evolve around instantaneous minima of the potential (see Fig. 6). This implies that the tensor to scalar ratio given in Eq. (42) is much smaller than one. In the numerical simulation of Fig. 2 for example, we obtain $r$ of the order $10^{-5}$ on cosmologically relevant scales. Hence this model satisfies the present observational upper bound: $r<0.3$ 3, 34. We also note that the spectral index $n_{T}$ of tensor perturbations is very close to scale-invariant.

Since the model (9) generally satisfies the relation $\epsilon \ll$ $\left|\eta_{\psi \psi}\right|$ during inflation except for the vicinity at $\eta_{\psi \psi}=0$, the spectral index (39) of scalar perturbations yields

$$
n_{\mathcal{R}} \simeq 1+2 \eta_{\psi \psi} \text {. }
$$

It is important to note that $n_{\mathcal{R}}$ is determined by $\eta_{\psi \psi}$ instead of $\eta_{\phi \phi}$. This reflects the fact that the background trajectory is not along the $\phi$ direction but along the $\psi$ direction.

The expression (43) is valid as long as the slow-roll parameter $\eta_{\psi \psi}$ is smaller than the order of unity. In the case of Fig. 5 for example, this can be used for $\eta_{\psi \psi}<0.2$ 0.3 after the fields almost stop oscillating and enter the inflationary stage. We have also computed the correlation measure $r_{c}$ numerically and have confirmed that $r_{c}$ is very small for the modes that crossed the Hubble radius during slow-roll inflation so that the expression (43) is valid.

In Fig. 7 we plot $n_{\mathcal{R}}$ in terms of the function of the number of e-foldings $N$ from the end of inflation for several different cases. For larger $D_{0}$ the total number of efoldings increases, which leads to the change of the curves from (a) to (c). The curve (b) corresponds to the case in which the number of e-foldings exceeds the typical COBE scale value $N=60$, while in the case of the curve (a) it does not reach cosmologically relevant scales. In the case (b) we obtain the value $n_{\mathcal{R}} \simeq 1.6$ for $N=60$, which is too large to satisfy recent observational constraints: $n_{\mathcal{R}}=0.97-1.21[35]{ }^{1}$

It is possible to realize the red-tilted spectrum $\left(n_{\mathcal{R}}<\right.$ 1) that satisfies observational constraints if cosmologically relevant scales are in the negative $\eta_{\psi \psi}$ region. In the case (c) of Fig. 7 for example, we obtain $n_{\mathcal{R}} \simeq 0.98$ for $N=60$. It is clear from Fig. 7 that the spectral indices around $N=60$ get smaller for larger total number of e-foldings.

\footnotetext{
${ }^{1}$ Without the running of the spectral index the constraint on $n_{\mathcal{R}}$ is severer: $n_{\mathcal{R}}=0.93-1.01$.
} 


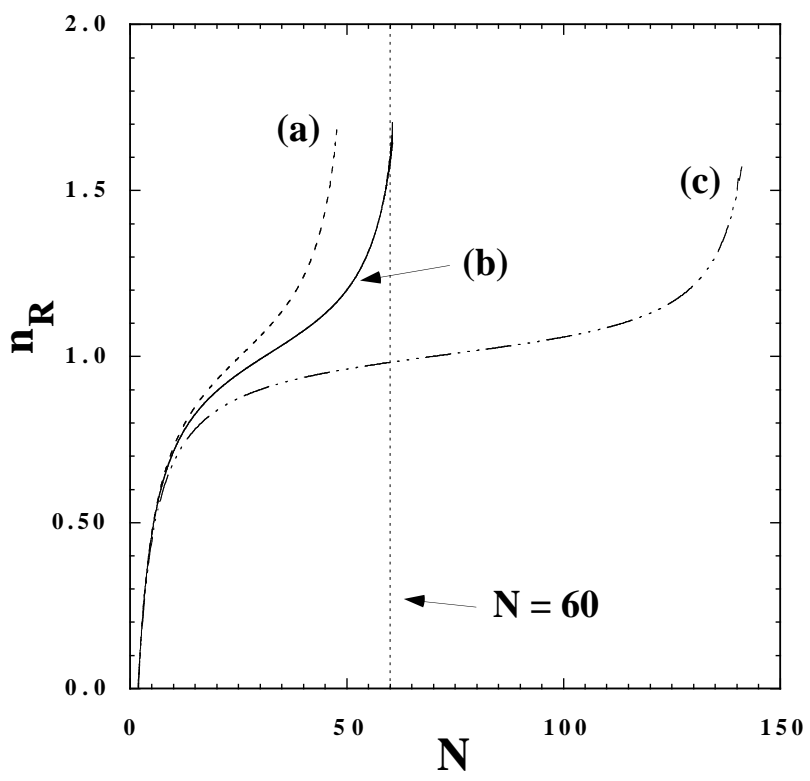

FIG. 7: The spectral index $n_{\mathcal{R}}$ as a function of the number of e-foldings $N$ from the end of inflation. Each case corresponds to (a) $D_{0}=1.213 \times 10^{-4}$, (b) $D_{0}=1.215 \times 10^{-4}$, and (c) $D_{0}=1.218 \times 10^{-4}$. Other model parameters are the same as in Fig. 2 .

The model also needs to satisfy the condition of the COBE normalization [ [3]

$$
\mathcal{P}_{\mathcal{R}} \simeq 2.4 \times 10^{-9}
$$

on cosmologically relevant scales observed by COBE. In Fig. 8 we find that in the case (b) this condition can be satisfied around the scale $N=60$, while the spectral index $n_{\mathcal{R}}$ is larger than observationally allowed values. We obtain the smaller $n_{\mathcal{R}}$ consistent with observations in the case (c), but the amplitude $\mathcal{P}_{\mathcal{R}}$ becomes much larger than the value (44). The increase of the amplitude reflects the fact that for larger total number of e-foldings the fields are stuck around instantaneous minima of the potential for a longer time so that $\epsilon$ tends to be smaller in Eq. (38). Thus for the model parameters chosen in Figs. 7 and 8 the model does not satisfy, simultaneously, both observational constraints of $n_{\mathcal{R}}$ and $\mathcal{P}_{\mathcal{R}}$.

We have tried many other cases and have not found a case in which both $n_{\mathcal{R}}$ and $\mathcal{P}_{\mathcal{R}}$ satisfy observational constraints. The behavior shown in Figs. 7 and 8 is typical in our model. In order to obtain a nearly scale-invariant spectrum, we need to adjust that cosmologically relevant scales $(N \sim 60)$ exist in the region around the maximum of the power spectrum. In this case, however, the amplitude $\mathcal{P}_{\mathcal{R}}$ tends to be too large to satisfy the COBE normalization. Nevertheless we have not searched for all parameter spaces; presumably there may be some regions or isolated points in the parameter space that satisfy both constraints of $n_{\mathcal{R}}$ and $\mathcal{P}_{\mathcal{R}}$ simultaneously. However the difficulty to find such viable parameters implies that the model requires severe fine-tunings in order to be consis-

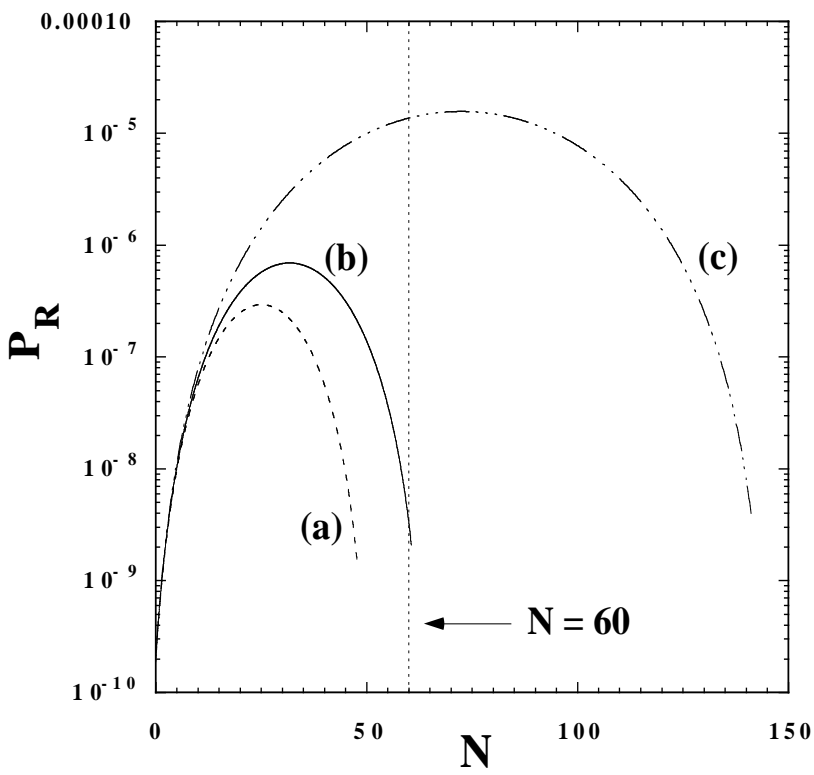

FIG. 8: The amplitude of the power spectrum $\mathcal{P}_{\mathcal{R}}$ as a function of the number of e-foldings $N$ from the end of inflation. Each case corresponds to (a) $D_{0}=1.213 \times 10^{-4}$, (b) $D_{0}=1.215 \times 10^{-4}$, and (c) $D_{0}=1.218 \times 10^{-4}$. Other model parameters are the same as in Fig. 2

tent with observations.

\section{CONCLUSIONS}

In this paper we have studied inflation in "delicate" Dbrane cosmology [17, 18] that takes into account effects of the moduli stabilization. The presence of the D3-brane in a warped conifold background gives rise to a correction to the warped four cycle volume, which leads to a modification to the nonperturbative superpotential $W$. Hence the total potential $V$ depends upon not only the inflaton field $\phi$ but also the (canonical) volume modulus field $\chi$.

Since the field $\chi$ evolves in two-field space even if the change is small, we need to study the dynamics of multifield inflation in an appropriate way. If the mass of $\chi$ is much larger than the Hubble parameter, this field quickly evolves toward instantaneous minima of the potential. In Ref. [17, 18, 19] the approximate relation (20) with respect to the non-canonical field $\sigma=\exp \left(\sqrt{2 / 3} \chi / M_{\mathrm{pl}}\right)$ was derived under the condition $V_{, \sigma}=0$. This relation tends to be accurate for smaller $\phi$, as we have shown in Fig. 11 The effective single field potential in terms of $\phi$ can be obtained by substituting Eq. (20) for the twofield potential (9). Numerically we have found that this description underestimates the total number of e-foldings even by one order of magnitude, see Fig. [4

This discrepancy comes from the fact that the actual background trajectory is not given by the effective single field $\phi$ but by the field $\psi$ defined by Eq. (22). If the 
scalar fields evolve along a straight line with constant $\chi$ during inflation, as in the case of hybrid inflation with the potential $V=\frac{\lambda}{4}\left(\chi^{2}-M^{2} / \lambda\right)^{2}+\frac{1}{2} g^{2} \phi^{2} \chi^{2}+\frac{1}{2} m^{2} \phi^{2}$, the single-field description with respect to $\phi$ is valid because the $\psi$ direction coincides with the $\phi$ direction. If the trajectory is curved as in the model (9), one has to study the dynamics of inflation along the $\psi$ direction. In fact in the numerical simulation of Fig. 5 the period of inflation $\left(0.3<\phi / \phi_{\mu}<0.5\right)$ coincides with the one derived by the slow-roll condition $\left|\eta_{\psi \psi}\right|<1$, but the slow-roll parameter $\eta_{\phi \phi}$ is larger than unity during this epoch.

We also find that the total number of e-foldings is very sensitive to a slight change of model parameters. This is related to the fact that the naive single $\phi$ field description grossly underestimates the total amount of inflation. Although the change of the field $\chi$ during inflation is typically small, the evolution of the volume modulus nontrivially affects the dynamics of inflation. It is interesting to note that in two-field model (9) one can realize a large number of e-foldings to solve horizon and flatness problems even if it is difficult in single-field context.

We have also evaluated the power spectra of density perturbations generated in this model. In two-field models of inflation there exist isocurvature perturbations in addition to curvature perturbations in general. When one of the fields is much heavier than the Hubble rate, the correlation between curvature and isocurvature perturbations is generally very small. In this case the power spectrum $\mathcal{P}_{\mathcal{R}}$ of scalar metric perturbation is estimated by Eq. (38) together with the spectral index $n_{\mathcal{R}}$ given by Eq. (43). It is important to recognize that the spectral index is determined by the slow-roll parameter $\eta_{\psi \psi}$ instead of $\eta_{\phi \phi}$. We have found that it is generally difficult to satisfy observational constraints of both the spectral index and the COBE normalization simultane- ously. This comes from the fact that as the spectrum approaches scale-invariant $\left(n_{\mathcal{R}}=1\right)$ the amplitude $\mathcal{P}_{\mathcal{R}}$ tends to be larger than the COBE normalized value $\left(\mathcal{P}_{\mathcal{R}} \simeq 2.4 \times 10^{-9}\right)$ on cosmologically relevant scales, see Figs. 7 and 8

The model we have studied is in fact "delicate" and needs severe fine-tunings of model parameters to satisfy several constraints discussed in this paper. The implementation of corrections due to the D3-brane motion to multi-throat D-brane models [36] can possibly improve the situation. It is to be noted that a viable model of inflation should be followed by a successful reheating 37. which is a challenging problem for D-brane cosmology at present. The multi-throat models have recently given some hopes in this direction, see Refs. [38] on the related theme. These are important issues of string cosmology which require further investigation.

\section{ACKNOWLEDGMENTS}

We thank D. Baumann, A. Dymarsky, I. Klebanov, L. McAllister, E. Pajer and I. Thongkool for useful discussions and clarifications. S.P. and M.S. thank JSPS for financial supports for their visits to Japan and for kind hospitality in Gunma National College of Technology, Nagoya University and Tokyo Institute of Technology. S.P. is supported by DST/JSPS (Grant No. DST/INT/JSPS/Proj-35/2007). M. S. is supported by DST/JSPS (Grant No. DST/INT/JSPS/Proj35/2007), JSPS fellowship (FY2007) and by ICTP and IUCAA through their associateship programs. S.T. is supported by JSPS (Grant No. 30318802).
[1] A. A. Starobinsky, Phys. Lett. B 91 (1980) 99; D. Kazanas, Astrophys. J. 241 L59 (1980); K. Sato, Mon. Not. R. Astron. Soc. 195, 467 (1981); A. H. Guth, Phys. Rev. D 23, 347 (1981).

[2] D. N. Spergel et al., AstroAphys. J. Suppl. 148, $175(2003)$.

[3] D. N. Spergel et al., arXiv:astro-ph/0603452

[4] J. Polchinski, Phys. Rev. Lett. 75, 4724 (1995).

[5] A. Sen, Remarks on Tachyon Driven Cosmology; arXiv:hep-th/0312153 and references therein.

[6] A. D. Linde, Inflationary Cosmology, arXiv:0705.0164 [hep-th].

[7] R. Kallosh, Inflation in string theory, arXiv:hep-th/0702059.

[8] G. R. Dvali and S. H. H. Tye, Phys. Lett. B 450, 72 (1999); G. R. Dvali, Q. Shafi and S. Solganik, arXiv:hep-th/0105203; C. P. Burgess et al., JHEP 0107, 047 (2001); G. Shiu and S. H. Tye, Phy. Lett. B 516, 421(2001); S. Alexander, Phys. Rev.D 65, 023507(2002); J. Garcia-Bellido, R. Rabadan and F. Zamora, JHEP 0201, 036 (2002); M. Gomez-Reino and I. Zavala, JHEP 0209, 020 (2002); C. P. Burgess, et al., JHEP 0203, 052(2002); N. T. Jones, H. Stoica and S. H. H. Tye, JHEP 0207, 051 (2002); D. Choudhury, D. Ghoshal, D. P. Jatkar and S. Panda, JCAP 0307, 009 (2003).

[9] S. Thomas and J. Ward, Phys. Rev. D 72, 083519(2005); S. Panda, M. Sami, S. Tsujikawa, Phys. Rev. D 73, 023515(2006); S. Panda, M. Sami, S. Tsujikawa and J. Ward, Phys. Rev. D 73, 083512 (2006); B. Gumjudpai, T. Naskar and J. Ward, JCAP 0611, 006 (2006); E. Papantonopoulos, I. Pappa and V. Zamarias, JHEP 0605, 038 (2006); A. Sen, arXiv:hep-th/0703157

[10] S. B. Giddings, S. Kachru and J. Polchinski, Phys. Rev. D 66, 106006 (2002).

[11] I. R. Klebanov and M. J. Strassler, JHEP 0008, 052 (2000).

[12] S. Kachru, R. Kallosh, A. Linde and S. P. Trivedi, Phys. Rev. D 68, 046005 (2003).

[13] S. Kachru, R. Kallosh, A. Linde, J. M. Maldacena, L. McAllister and S. P. Trivedi, JCAP 0310, 013 (2003).

[14] J. P. Hsu, R. Kallosh and S. Prokushkin, JCAP 0312, 
009 (2003); H. Firouzjahi and S. H. H. Tye, Phys. Lett. B 584, 147 (2004); C. P. Burgess, J. M. Cline, H. Stoica and F. Quevedo, JHEP 0409, 033 (2004); J. J. Blanco-Pillado et al., JHEP 0411, 063 (2004); JHEP 0609, 002 (2006); F. Koyama, Y. Tachikawa and T. Watari, Phys. Rev. D 69, 106001 (2004); J. M. Cline and H. Stoica, Phys. Rev. D 72, 126004 (2005); R. Allahverdi, K. Enqvist, J. Garcia-Bellido and A. Mazumdar, Phys. Rev. Lett. 97, 191304 (2006); R. Allahverdi et al., JCAP 0706, 019 (2007).

[15] D. Baumann, A. Dymarsky, I. R. Klebanov, J. Maldacena, L. McAllister and A. Murugan, JHEP 0611, 031 (2006).

[16] S. Kuperstein, JHEP 0503, 014 (2005).

[17] D. Baumann, A. Dymarsky, I. R. Klebanov, L. McAllister and P. J. Steinhardt, arXiv:0705.3837 [hep-th].

[18] D. Baumann, A. Dymarsky, I. R. Klebanov and L. McAllister, arXiv:0706.0360 [hep-th].

[19] A. Krause and E. Pajer, arXiv:0705.4682 [hep-th].

[20] O. DeWolfe and S. B. Giddings, Phys. Rev. D 67, 066008 (2003).

[21] M. Berg, M. Haack and B. Kors, Phys. Rev. D 71 (2005) 026005; arXiv:hep-th/0409282

[22] S. Gukov, C. Vafa and E. Witten, Nucl. Phys. B 584, 69 (2000) [Erratum-ibid. B 608, 477 (2001)].

[23] S. B. Giddings and A. Maharana, Phys. Rev. D 73,126003 (2006).

[24] P. Koerber and L. Martucci, arXiv:0707.1038 [hep-th].

[25] A. D. Linde, Phys. Rev. D 49, 748 (1994).

[26] C. Gordon, D. Wands, B. A. Bassett and R. Maartens, Phys. Rev. D 63, 023506 (2001).

[27] D. Polarski and A. A. Starobinsky, Phys. Rev. D 50, 6123 (1994); A. A. Starobinsky and J. Yokoyama, arXiv:gr-qc/9502002 J. Garcia-Bellido and D. Wands,
Phys. Rev. D 53, 5437 (1996); A. D. Linde and V. Mukhanov, Phys. Rev. D 56, 535 (1997); D. Langlois, Phys. Rev. D 59, 123512 (1999); A. A. Starobinsky, S. Tsujikawa and J. Yokoyama, Nucl. Phys. B 610, 383 (2001).

[28] H. Kodama and M. Sasaki, Prog. Theor. Phys. Suppl. 78, 1 (1984).

[29] B. A. Bassett, S. Tsujikawa and D. Wands, Rev. Mod. Phys. 78, 537 (2006).

[30] D. Wands, N. Bartolo, S. Matarrese and A. Riotto, Phys. Rev. D 66, 043520 (2002).

[31] C. T. Byrnes and D. Wands, Phys. Rev. D 74, 043529 (2006).

[32] S. Tsujikawa, D. Parkinson and B. A. Bassett, Phys. Rev. D 67, 083516 (2003); D. Parkinson, S. Tsujikawa, B. A. Bassett and L. Amendola, Phys. Rev. D 71, 063524 (2005).

[33] N. Bartolo, S. Matarrese and A. Riotto, Phys. Rev. D 64, 123504 (2001).

[34] M. Tegmark et al., Phys. Rev. D 74, 123507 (2006).

[35] W. H. Kinney, E. W. Kolb, A. Melchiorri and A. Riotto, Phys. Rev. D 74, 023502 (2006).

[36] N. Iizuka and S. P. Trivedi, Phys. Rev. D 70, 043519 (2004).

[37] L. Kofman, L. D. Linde and A. Starobinsky, Phys. Rev. D 56, 3258 (1997).

[38] N. Barnaby, C. P. Burgess and J. M. Cline, JCAP 0504, 007 (2005); L. Kofman and P. Yi, Phys. Rev. D72, 106001 (2005); D. Chialva, G. Shiu and B. Underwood, JHEP 0601, 014 (2006); J. H. Brodie and D. A. Easson, JCAP 0312, 004 (2003); A. R. Frey, A. Mazumdar and R. Myers, Phys. Rev. D 73, 026003(2006); S. Mukohyama, arXiv:0706.3214 [hep-th]. 